\title{
Evaluating the Influence of Taxi Subsidy Programs on Mitigating Difficulty Getting a Taxi in Basis of Taxi Empty-loaded Rate
}

\author{
Jialin Wen ${ }^{1}$, Min Zou ${ }^{2}$, Yikai Ma ${ }^{3}, \&$ Hao Luo ${ }^{4}$ \\ ${ }^{1}$ Electrical and Information College, Jinan University, China \\ Correspondence: Jialin Wen, Electrical and Information College, Jinan University, Qianshan Road 206, Zhuhai, China. \\ E-mail:1094886064@qq.com
}

Received: November 30, 2016

Accepted: January 5, 2017 Online Published: February 13, 2017

doi:10.5539/ijsp.v6n2p9

URL: https://doi.org/10.5539/ijsp.v6n2p9

\begin{abstract}
With the advent of the "Internet plus" era, a number of companies have established the service platform of taxi-hailing apps relying on the mobile Internet, which builds up a communication bridge between passengers and taxi drivers. Besides, taxi companies have initiated many subsidy programs. Based on the prediction model of passenger waiting time built in this paper, it has been proved that there exists a negative correlation between passenger waiting time and taxi empty-loaded rate. This paper also analyzes the influencing factors of taxi empty-loaded rate. The results show that the higher the taxi sharing rate is, the lower the taxi empty-loaded rate is. And the longer the average operation time is, the higher the taxi empty-loaded rate is. By comparing various taxi subsidy programs, this paper finally draws a conclusion that it will be much more difficult to take a taxi if taxi companies provide subsidies for passengers. But the difficulty in taking a taxi can be alleviated if taxi companies provide subsidies for taxi drivers.
\end{abstract}

Keywords: prediction model of passenger waiting time, arrival time interval of empty taxis

\section{Introduction}

How to alleviate the difficulty in taking a taxi is an important issue facing urban traffic. Nowadays, most taxis can provide round-the-clock service. Although the number of taxis has been increasing, the difficulty in taking a taxi can not be resolved ultimately. Even worse, the operating efficiency of taxis has been declining. The increase of urban population and diversification of social activities make the demand for taxi rise. As the traffic jam becomes worse in cities, to guarantee personal income, many taxi drivers are unwilling to drive on busy roads and main urban zones, which makes it hard for many citizens to take a taxi near some transportation junctions, commercial centers and hospitals. City construction affects the efficiency of taxi. Not being standard in the taxi industry and taxi drivers' refusal to take passengers are the human factors that make it difficult to take a taxi. The most serious disadvantage of those factors is the extremely high empty-loaded rate---some taxi drivers drive aimlessly. And passengers are confused about the fact that it's impossible to take a taxi on rainy days or during rush hours even though they have been waiting for a long time.

With the development of "Internet plus" era, the taxi-hailing app Didi is becoming more and more popular among people. The unique ad-vantages of Didi have a profound effect on traditional taxi industry. The-se taxi-hailing apps always have their typical subsidy programs. The subsidy war between Didi and Kuaidi has drawn public attention. They have lost billions of dollars subsidizing riders and drivers in a bid for market share. This paper aims to discover what influence these subsidy programs have made on easing the difficulty in taking a taxi.

At present, Lu Shufang and other scholars have found that taxi-hailing apps like Didi can be great of great assistance for easing the difficulty in taking a taxi. Qi Guande and other scholars have predicted the passenger waiting time. Cao Yi and other scholars have searched for the influence of usage rate of taxi-hailing apps on urban taxi social welfare. Wang Guangtao and other scholars have made suggestions about integrating the management of taxi-hailing apps. Scholar Wu Yonghua has made the existing problem and countermeasure analysis of taxi-hailing apps. Zhang Zhenzhen has pointed out that the underlying cause of the difficulty in taking a taxi lies in the taxi company system, and high taxi rental fees lead to taxi drivers' unwillingness to take passengers during rush hours or shift time. AnYukang has researched on the difficulty in taking a taxi in Beijing area from the point of supply and demand. Sun Huitai has studied the strategies of alleviating the difficulty in taking a taxi, such as increasing effective supply of taxis and diverting the demand of taking a taxi. Geng Yonghao has expected to alleviate the difficulty in taking a taxi by presenting taxi drivers with gasoline cards if they can take passengers to the designed places. Jiang Yuan has made the conclusion that the extension of the city scale would increasingly deepen the difficulty in taking a taxi. 
The difficulty in taking a taxi means that it's hard for passengers to take a taxi in the expected time or there is no taxi available. What's more, some taxi drivers are picky, meddlesome and even refuse to take passengers. The concentrated expression of the difficulty in taking a taxi is passenger's long waiting time. If the subsidy programs really decrease the passenger's waiting time to a certain extent, it can prove that subsidy programs can resolve the difficulty in taking a taxi.

Passenger waiting time is closely related to empty-loaded rate, so this paper attempts to find out the relationship between passenger waiting time and empty-loaded rate. Many factors contribute to the empty-loaded rate. By way of the study on the major factors influencing the empty-loaded rate, the researcher is able to get the interaction between taxi subsidy programs and empty-loaded rate. And then the researcher deduces the relationship of passenger waiting time and taxi subsidy programs. Namely, the relationship between the degree of difficulty in taking a taxi and taxi subsidy programs can be obtained. Finally, this paper examines whether the implementation of subsidy programs is helpful to alleviate the difficulty in taking a taxi.

\section{Evaluation of the Model}

The difficulty in taking a taxi means that it's hard for passengers to take a taxi in the expected time or there is no taxi available. What's more, some taxi drivers are picky, meddlesome and even refuse to take passengers. The concentrated expression of the difficulty in taking a taxi is passenger's long waiting time. If the subsidy programs really decrease the passenger's waiting time to a certain extent, it can prove that subsidy programs can resolve the difficulty in taking a taxi. Next this paper will discuss the relationship between subsidy programs and passenger waiting time.

\subsection{The Prediction Model of Passenger Waiting Time}

The high-pressured water jet cutting is a special way of removal. The water jet could reach the pressure up from 10MPa to $400 \mathrm{MPa}$ or even higher with the help of superchargers. The water gains pressure energy and squirts out of tiny injectors, then the pressure energy transforms into kinetic energy therefore forms a high speed jet-flow. The water jet removal reaches its goal of removing the debris by using this kinetic energy of the high speed jet-flow to shock and do damage to the workpieces.

\subsubsection{Modelling of the Arrival Time Intervals of Empty-loaded Car}

This paper uses the exponential distribution method to model the arrival time intervals of empty-loaded car. With the course of time, the arrival quantity of the empty-loaded car in a certain place is a classic Poisson process. In time divisions, the arrival quantity of the empty-loaded car in a certain place obeys Poisson distribution, namely the arrival time intervals should obey exponential distribution. As the increasing rate of the arrival quantity of the empty-loaded car in a certain place varies in real life, so does the arrival time interval, the paper works on the assumption that the arrival time interval of empty-loaded car in a specified time period of obeys the time-sharing exponential distribution. In this situation, the rate of empty-loaded car is inversely proportional to the arrival time interval. Figure 1 and Figure 2 visualize the relation between the arrival quantity of the empty-loaded car in a certain place and exponential distribution in a period of time.

Since the quantity of empty taxis is nearly not affected by the surrounding environment, this paper holds that the event of the taxi driver arriving at some place is ran-dom. Then the probability distribution table is built based on Poisson distribution.

Table 1. The Probability Distribution Table Based on Poisson Distribution

\begin{tabular}{llllllllllllll}
\hline $\mathrm{P}_{1}(\mathrm{t})$ & $\mathrm{P}_{2}(\mathrm{t})$ & $\mathrm{P}_{3}(\mathrm{t})$ & $\mathrm{P}_{4}(\mathrm{t})$ & $\mathrm{P}_{5}(\mathrm{t})$ & $\mathrm{P}_{6}(\mathrm{t})$ & $\mathrm{P}_{7}(\mathrm{t})$ & $\mathrm{P}_{8}(\mathrm{t})$ & $\mathrm{P}_{9}(\mathrm{t})$ & $\mathrm{P}_{10}(\mathrm{t})$ & $\mathrm{P}_{11}(\mathrm{t})$ & $\mathrm{P}_{12}(\mathrm{t})$ & $\mathrm{P}_{13}(\mathrm{t})$ & $\mathrm{P}_{14}(\mathrm{t})$ \\
\hline 0.015 & 0.034 & 0.061 & 0.091 & 0.117 & 0.132 & 0.132 & 0.119 & 0.093 & 0.071 & 0.047 & 0.030 & 0.007 & 0.004 \\
\hline
\end{tabular}




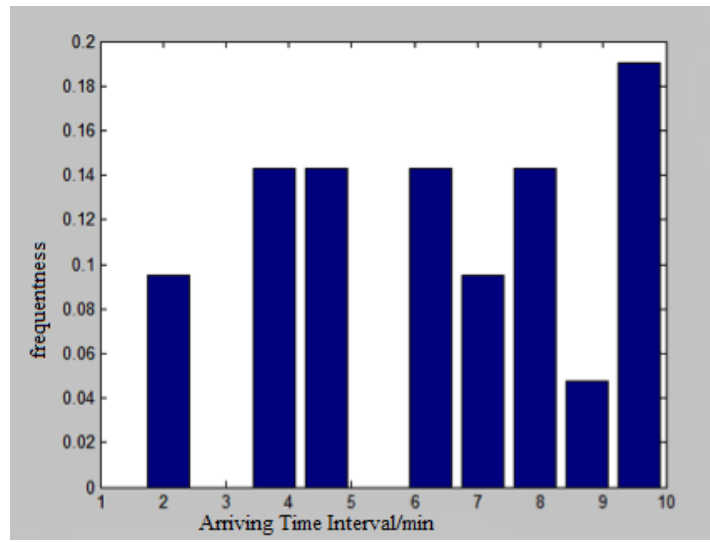

Figure 1. The Distribution of Arriving Time Interval between 12:00-13:00 in Guangzhou

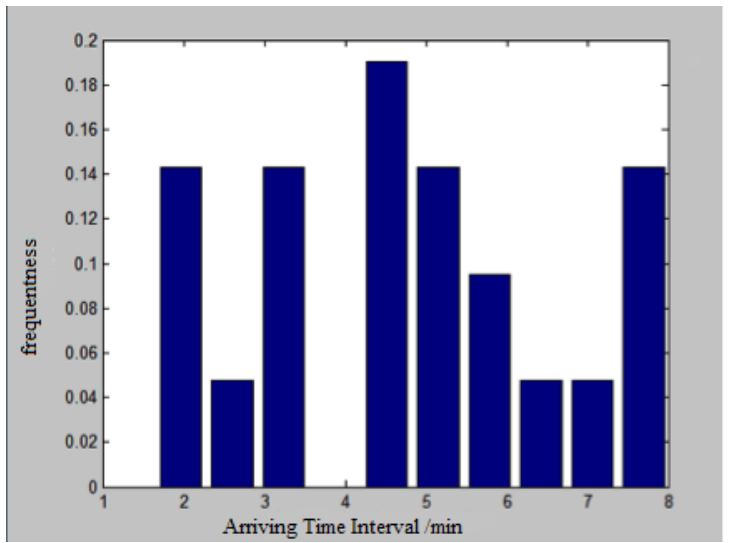

Figure 2.The Actual Distribution of Waiting Time between 12:00-13:00 in Guangzhou

\subsubsection{Passenger Waiting Time Modelling}

This paper calculates the passenger waiting time distribution by estimating the worst case. Firstly, this paper assumes that passengers arrive and wait for taxis uniformly, randomly and equiprobably within a specified time period of $t$. As a result, it is suggested that the probability density where passengers are in the time period of $t$ is proportionate to the product $p(t) t$ of the probability density of time interval itself and the length of the time interval. Next, a passenger needs to wait for a period of $t$ to get on a taxi at most in the worst case when he is in the time interval of $t$. Thus in the worst case, the probability density $f(\Delta t)$ of waiting time $W T=t$ is in proportion to $p(t) t$, normalized to the formula:

$$
f(W T=t)=\frac{P(t) t}{\int_{0}^{\infty} P(t) t d t}=\frac{\lambda e^{-\lambda t} t}{\int_{0}^{\infty} \lambda e^{-\lambda t} t d t}=\lambda^{2} e^{-\lambda t} t
$$

In this formula, $p(t) t$ stands for the product of the probability density of time interval itself and the length of the time interval. $p(t)$ stands for the probability density of time interval itself, $\lambda$ stands for simple variable relating waiting time and probability of getting on the taxi.

Base on the the waiting time distribution(formula(1)), the cumulative probability of getting on the taxi $F(T)=P(W T \leq T)$ is calculated by the distribution of cumulative probability.

$$
F(T)=\int_{0}^{T} f(t) d t=\int_{0}^{T} \lambda^{2} e^{-\lambda t} d=1-\lambda T e^{-\lambda T}-e^{-\lambda T}
$$

$\lambda$ refers to a single variable of passenger waiting time and the probability of taking taxis, $W T=t$ refers to the passenger waiting time, $W T=t$ refers to the cumulative probability of taking taxis when passengers have waited for some time. 


\subsubsection{The Model Parameters}

The parameter $\lambda$ As for a period of time, the passenger waiting time is a random variable. Its probability density and cumulative distribution can be calculated by parameter estimation by historical data. According to formula 2 , the relationship between the passenger waiting time and the probability of taking taxis is only associated with the single variable $\lambda$. So this paper adopts maximum likelihood estimation.

$$
\bar{\lambda}=\arg \max \bar{l}\left(\lambda \mid t_{1}, \ldots, t_{n}\right)=\arg \max _{\lambda} \lambda^{n} e^{-\lambda \sum t_{i}}=\sum t_{i} / n=\frac{1}{\mu}
$$

$t_{1} \ldots t_{n}$ is the time interval of the empty-loaded cars' arriving time, $\mu$ is the sample mean of arrival time interval.

The length of time: The length of time has an influence on the actual time interval for arriving of empty taxi and exponential distribution fitting. The best fitting effect is the length of one hour. As shown in figure 3 , when the length of time is too short, the quantity of empty arriving taxis will be small, and the distribution of time interval for arriving of empty taxi will be more easily affected by the environment, and the fitting effect will become worse. When the length of time is too long, time interval for arriving of empty taxi may be variant in this period, and the fitting effect will become worse, too. Therefore, this paper assign one hour as time length's value.

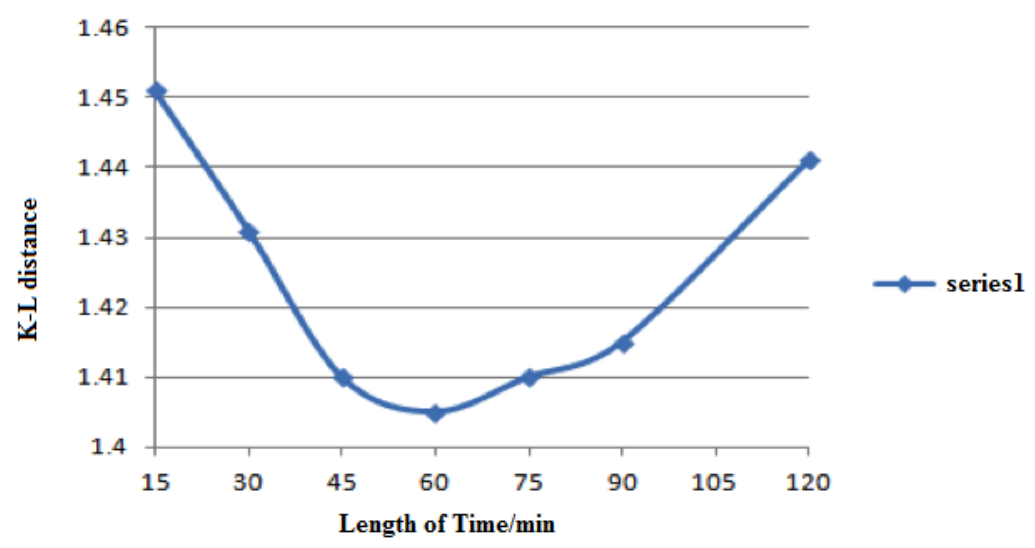

Figure 3. The Effect of Length of Time on the Result of Exponential Distribution Fitting

On the premise that an hour is assigned as the value of time length, the value distribution of $\lambda$ is shown in table 2 . 
Table 2. The Value of $\lambda$ when the Length of Time is an Hour

\begin{tabular}{|c|c|}
\hline the Length of Time & $\lambda_{i}(i=1,2 \ldots 14)$ \\
\hline 7:00 8:00 & 0.15 \\
\hline 8:00 9:00 & 0.12 \\
\hline 9:00 10:00 & 0.14 \\
\hline $10: 00 \sim 11: 00$ & 0.18 \\
\hline $11: 00 \sim 12: 00$ & 0.16 \\
\hline $12: 00 \sim 13: 00$ & 0.15 \\
\hline $13: 00 \sim 14: 00$ & 0.14 \\
\hline $14: 00 \sim 15: 00$ & 0.13 \\
\hline $15: 00 \sim 16: 00$ & 0.17 \\
\hline $16: 00 \sim 17: 00$ & 0.21 \\
\hline 17:00 18:00 & 0.15 \\
\hline $18: 00 \sim 19: 00$ & 0.14 \\
\hline 19:00 20:00 & 0.2 \\
\hline 20:00 21:00 & 0.19 \\
\hline
\end{tabular}

When normalized value $\lambda$ is put into the model of empty intervals and passenger waiting time, two functions of time $t$ can be obtained.And because empty intervals negatively related to empty-loading ratio, passenger waiting time and taxi empty-loading ratio are fitted with matlab. Then the figure of some function of passenger waiting time and empty-loading ratio can be obtained.

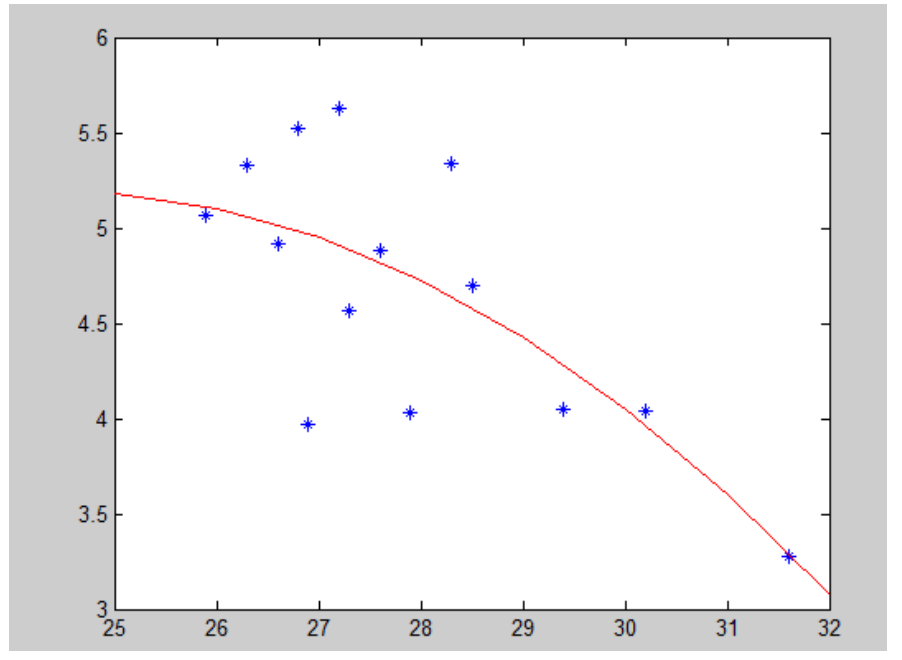

Figure 4. Passenger Waiting time and Empty-loading Ratio

As the figure shows, the horizontal axis demonstrates the taxi empty-loaded rate and the vertical axis demonstrates the passenger waiting time. 
Judging from the figure, it is not difficult to draw the conclusion that the higher the taxi empty-loaded rate is, the shorter the passenger waiting time is. This conclusion is in conformity with the assumption which was made according to the modelling study. Thus, it is reasonable to deduce the passenger waiting time from empty-loaded rate. In other words, the taxi empty-loaded rate is inversely proportional to the passenger waiting time.

\subsection{Analysis on Major Influencing Factors of the Empty-loaded Rate}

Urban resident trip survey plays a central role in urban traffic planning. On the basis of urban taxi traffic mode survey and taxi operating status survey, this paper analyzes empty-loaded rate of urban taxi.

\subsubsection{The Turnover Volume of Urban Resident Travel By Taxi}

The turnover volume of urban resident travel by taxi are products of the passenger capacity and the distance traveled by the taxi.

$$
Z=P \cdot Q \cdot R \cdot D=P \cdot Q \cdot R \cdot\left(S+\frac{C-m_{0}}{m}\right)
$$

In the formula (4), Zrefers to the turnover volume of urban resident travel by taxi.Prefers to the total number of urban citizens. $Q$ refers to the rate of urban resident trip (time/day). $R$ refers to the sharing rate of urban taxi traffic mode. $D$ refers to the average trip distance $(\mathrm{km}) . C$ refers to the average cost of residents in taking a taxi (yuan). $m_{0}$ refers to the flag-down fare(yuan). $S$ refers to the starting mileage $(\mathrm{km}) . m$ refers to the charging fee per kilometer (yuan/km).

\subsubsection{Total Mileage of Occupied Taxi Every Day}

The number of the passengers is variable each time when the taxi is in service. Thus average number of the passengers each time is used to calculate the total mileage of occupied taxi every day, the formula is:

$$
L=\frac{Z}{A}
$$

In the formula (5), $L$ refers to the total mileage per day $(\mathrm{km}) . A$ refers to the average number of passengers taking taxis (ten thousand people/a taxi).

\subsubsection{Calculation of the Taxi Empty-loaded Rate}

The Formula of the Taxi Empty-loaded Rate is as follows.

$$
K=1-\frac{L}{T \bar{V} n}
$$

In formula (6), $K$ refers to the taxi empty-loaded rate(\%). $\bar{V}$ refers to the average service time per day $(\mathrm{km} / \mathrm{h}), n$ refers to the taxi quantity.

In the formula (6), the taxi quantity can be obtained by calculating the taxi quantity. Considering the temporal distribution of passenger source has obvious difference, the taxi quantity calculated by the rate of resident trip per day may exceed the demand of resident night trip because the demand of resident day trip is ignored. So it's needed to correct the formula (6).

$$
K=1-\frac{x L}{y T \bar{V} n}
$$

In the formula (7), $x, y$ stands for the quantity travel by taxi by percentage of $x$ in a period of $y T$. Substitute formula (5) and (6) in formula (4).

$$
K=1-\frac{x P Q}{A f y T \bar{V}} \cdot\left(S+\frac{c-m_{0}}{m}\right) \cdot \frac{R}{N}
$$

\subsubsection{Analysis on the Major Influencing Factors of the Taxi Empty-loaded Rate}

In order to analyze the influencing factors of taxi quantity,

$$
\frac{x P Q}{A f y T \bar{V}} \cdot\left(S+\frac{c-m_{0}}{m}\right)=g
$$

The relational model among taxi quantity, empty-loaded rate and sharing rate is established.

$$
K=1-\frac{g R}{N}
$$

The scale coefficient $\mathrm{g}$ is determined by the parameter of resident trip, taxi service, etc. It reflects the sensitivity level of 
the taxi empty-loaded rate to the sharing rate and taxi quantity. The bigger the value of $g$ is, the higher the sensitivity level of the taxi empty-loaded rate to the sharing rate and taxi quantity is. When the city develops into a certain stage, both of the economic level of residents and urban traffic structure tend to be stable and change a little in short time. Hence The scale coefficient $g$ may be considered as constant.

According to the formula (10), the empty-loaded rate of taxi is negatively related to sharing rate but positively related to taxi quantity.

\section{Analysis on Various Subsidy Programs}

\subsection{The Influence of Subsidy Programs on Alleviating the Difficulty in Taking a Taxi throughout the City}

The taxi subsidy programs of Kuaidi and Didi are shown in table 3 and table 4 respectively.

As can be seen from the table above, the subsidy programs of Didi and Kuaidi are mainly reflected in two aspects: subsidy on passengers and subsidy on taxi drivers. Meanwhile, subsidy programs directly affect the passenger's total fare of taxi and taxi driver's profitability.

With this subsidy, passengers can spend less money on taking taxis and they are more willing to taking taxis. Then the sharing rate of taxi will be increased. According to the formula $K=1-\frac{g R}{N}$, the decrease of the empty-loaded rate can lead to the increase of passenger waiting time.

In addition, taxi drivers can gain more profits with this subsidy. And they are more willing to picking up passengers. Thus the business hours of taxis are increased. Based on the formula $K=1-\frac{L}{T \bar{V} n}$, the increase of the empty-loaded rate can lead to the decrease of passenger waiting time.

From the perspective of whole city, it can be concluded that subsidy programs really decrease passenger waiting time, and then the difficulty in taking a taxi will be alleviated.

Table 3. The Changes of Subsidy Program of Kuaidi

\begin{tabular}{|c|c|}
\hline Date & The Change of Subsidy Program \\
\hline \multirow[t]{2}{*}{ 2014.1.20 } & Total fee is reduced by 10 yuan, \\
\hline & Driver gets a reward of 10 yuan. \\
\hline \multirow[t]{2}{*}{ 2014.2.17 } & Total fee is reduced by 10 yuan, \\
\hline & Driver gets a reward of 5-11 yuan. \\
\hline 2014.2.18 & Passenger gets 13 yuan as subsidy. \\
\hline \multirow[t]{2}{*}{2014.3 .4} & Passenger gets 10 yuan as subsidy, \\
\hline & Drivers' subsidy remains unchanged. \\
\hline 2014.3 .5 & Passenger gets 5 yuan as subsidy. \\
\hline 2014.3.22 & Passenger gets 3-5 yuan as subsidy. \\
\hline 2014.5.17 & Passenger gets no subsidy. \\
\hline 2014.7 .9 & Driver gets 2 yuan as subsidy. \\
\hline 2014.8 .9 & Driver gets no subsidy. \\
\hline
\end{tabular}


Table 4. The Changes of Subsidy Program of Didi

\begin{tabular}{cc}
\hline Date & The Change of Subsidy Program \\
\hline 2014.1 .10 & Total fee is reduced by 10 yuan, \\
& Driver gets a reward of 10 yuan. \\
2014.2.17 & Total fee is reduced by 10-15 yuan, \\
& Driver gets a reward of 50 yuan for the first business. \\
2014.2 .18 & Passenger gets 12-20 yuan as subsidy. \\
2014.3 .7 & Passenger gets 6-15 yuan as subsidy at random. \\
2014.3 .23 & Passenger gets 3-5 yuan as subsidy. \\
2014.5 .17 & Passenger gets no subsidy. \\
2014.7 .9 & Driver gets 2 yuan as subsidy. \\
2014.8 .12 & Driver gets no regular subsidy. \\
\hline
\end{tabular}

\subsection{The Influence of Subsidy Programs on Alleviating the Difficulty in Taking a Taxi during Rush Hours}

With this subsidy, taxi drivers can gain more profits and more incentives to work. Then the daily operating time of taxis will be increased because taxi drivers are more willing to work during rush hours. According to the formula $K=1-\frac{L}{T \bar{V} n}$, the increase of empty-loaded rate can lead to the decrease of passenger waiting time. Thus the difficulty in taking taxi during rush hours will be alleviated.

\subsection{The Influence of Subsidy Programs on Alleviating the Difficulty in Taking a Taxi in Congested Areas}

Subsidizing on passengers may deepen the difficulty in taking a taxi in congested areas. To some degree, such subsidy programs increase the number of passengers, and also increase the sharing rate of taxis. Then the rate of empty taxis will be lower and passenger waiting time will be longer. That is, this kind of subsidy programs can not alleviating the difficulty in taking a taxi in congested areas.

The increase of the taxi driver's operating time contributes to the empty-loaded rate, then the passenger waiting time will be decreased. Therefore, difficulty in taking a taxi in congested areas can be alleviated.

\section{Conclusion}

The number of empty taxis arriving at some area can be regarded as a classical Poisson process. Based on the prediction model of passenger waiting time built in this paper, it has been proved that there exists a positive correlation of passenger waiting time with empty taxi arrival time interval. And because taxi empty-loaded rate is negatively related to empty taxi arrival time interval, there exists a negative correlation between passenger waiting time and taxi empty-loaded rate.

This paper also analyzes the influencing factors of taxi empty-loaded rate. The results show that the higher the taxi sharing rate is, the lower the taxi empty-loaded rate is. And the longer the average operation time is, the higher the taxi empty-loaded rate is.

By comparing various taxi subsidy programs, this paper finally draws a conclusion that it will be much more difficult to take a taxi if taxi companies provide subsidies for passengers. But the difficulty in taking a taxi can be alleviated if taxi companies provide subsidies for taxi drivers. 


\section{References}

Lu, S. F., Zhou, J., \& Ren, Y. N. A (2014). Study on the Traffic Situation of "Difficulty in Get-ting A Car" and Its Countermeasures - "You Call Me" Instant Taxi[J].Man-agement\&Technolosy of SME, (01), 135-136.

Qi, G. D., Pan, Y., Li, S. J., \& Pan, G. (2013). Predicting passengers' waiting time by mining taxi traces. Ruan jian Xue Bao/Journal of Software, 24(Suppl.(2)), 14-23(inChinese). http://www.jos.org.cn/1000-9825/13019.htm

Cao Y, \& Luo, X. A. (2015). Forecasting Model of Trip Distribution for Vacant Taxis withTaxi-hailing Apps[J]. Journal of Wuhan University of Technology(Transportation Science\&Engineering), (01), 51-54+59.

Wang, G. T., Qi, T. Y., Chen, X. H., Yin, G. T., Li, Q., Lu, X. M., ..,\& Tan, Y. Z. (2014). Integrated Management of Taxi Booking Apps[J]. Urban Transport of China, (05), 1-3. https://doi.org/10.18686/utc.v1i1.1

Wu, Y. H. (2015). Analysis on the Problems and Countermeasures of Taxi Booking Apps [J]. China Market, (17), $102-103+112$.

Zhang, Z. Z. (2014). An Analysis of the Difficulty in Getting A Car[J]. CHINA-TODAY FORUM, (10), 1-3.

An, Y. K. (2013). Research on the Difficulty of Taxi Taxis in Beijing Based on the Relationship between Supply and Demand[J]. Modern Economic Information, (05), 306.

Sun, H. T., He, Y. J., Zou, P. S., Hu, K. P., \& Chen, L. Q. (2014). A Study on the Tactics of Relieving "Difficulty in Taxi" Based on the Relationship between Supply and Demand[J]. Transportation Enterprise Management, (10), 1-3.

Geng, Y. H., \& Zhang, H. T. (2015). The Research About Some Policies of Solving "Taxi Difficult"Problems in the City Center_-Taking Nanjing as an Example[J]. Architecture\&Cultural, (07), 139-140.

Jiang, Y. (2012), Behind the "Difficulty in Taxi" [J]. CHINA STATISTICS, (06), 25.

Kang, L.W. (2010). Based on FCD city taxi empty rate and the calculation of theownership[J]. Digital Technology and Application, (07), 81-82.

\section{Appendixes}

Table 1. Distribution of Taxis in Beijing From DidiCangqiong Intelligent Platform

\begin{tabular}{ccc}
\hline Longitude & Latitude & Numbers of Taxis \\
\hline 121.4788 & 31.2143 & 555 \\
121.5136 & 31.1883 & 179 \\
114.324 & 30.5454 & 128 \\
121.6109 & 38.9121 & 98 \\
116.4581 & 39.9692 & 87 \\
121.5518 & 38.9612 & 85 \\
116.4581 & 39.9603 & 80 \\
116.4953 & 39.9892 & 78 \\
116.5139 & 40.0137 & 75 \\
116.4983 & 39.9133 & 73 \\
116.4758 & 39.9187 & 71 \\
116.5195 & 40.0207 & 70 \\
118.1808 & 39.63 & 70 \\
116.5039 & 40.0064 & 70 \\
116.4678 & 39.8938 & 68 \\
116.4952 & 39.9941 & 68 \\
118.3014 & 35.1099 & 67 \\
116.4713 & 39.9772 & 67 \\
116.3834 & 39.988 & 65 \\
116.4791 & 39.9916 & 67 \\
& & 70 \\
\hline
\end{tabular}




\begin{tabular}{|c|c|c|}
\hline 116.4944 & 39.9916 & 65 \\
\hline 116.4824 & 39.982 & 64 \\
\hline 116.466 & 39.9553 & 64 \\
\hline 116.3783 & 39.9712 & 63 \\
\hline 116.5263 & 40.0188 & 62 \\
\hline 114.4792 & 38.0154 & 62 \\
\hline 130.2768 & 47.3452 & 62 \\
\hline 116.4697 & 39.9551 & 62 \\
\hline 116.454 & 39.9954 & 62 \\
\hline 116.473 & 39.9373 & 61 \\
\hline 116.3145 & 39.8997 & 61 \\
\hline 116.4774 & 39.9065 & 61 \\
\hline 116.4813 & 39.9816 & 60 \\
\hline 116.3829 & 39.95 & 59 \\
\hline 116.3263 & 39.9026 & 59 \\
\hline 116.4988 & 40.0009 & 59 \\
\hline 125.9585 & 41.7474 & 59 \\
\hline 116.4755 & 39.9186 & 58 \\
\hline 116.3621 & 39.9875 & 58 \\
\hline 116.5083 & 40.0128 & 58 \\
\hline 116.4771 & 39.8857 & 58 \\
\hline 116.4663 & 39.8902 & 57 \\
\hline 122.9956 & 41.1145 & 57 \\
\hline 116.4766 & 39.917 & 56 \\
\hline 116.2847 & 39.9037 & 56 \\
\hline 116.4732 & 39.9064 & 56 \\
\hline 116.4255 & 39.9897 & 55 \\
\hline 116.5319 & 40.0228 & 55 \\
\hline 116.4434 & 39.9475 & 54 \\
\hline 116.4411 & 39.9211 & 54 \\
\hline 116.422 & 39.9753 & 54 \\
\hline 116.3498 & 39.9064 & 53 \\
\hline 124.3983 & 43.1727 & 53 \\
\hline 116.4539 & 39.9657 & 53 \\
\hline 116.576 & 40.0463 & 53 \\
\hline 116.3624 & 39.9727 & 53 \\
\hline 116.4506 & 39.9377 & 52 \\
\hline 116.3032 & 39.9919 & 52 \\
\hline 116.4714 & 39.9541 & 52 \\
\hline 116.4717 & 39.9233 & 52 \\
\hline 116.2839 & 39.9274 & 52 \\
\hline 116.4358 & 39.9076 & 52 \\
\hline 116.5557 & 40.0358 & 52 \\
\hline
\end{tabular}




\begin{tabular}{|c|c|c|}
\hline 125.979 & 41.7459 & 52 \\
\hline 116.4243 & 39.9501 & 52 \\
\hline 116.4354 & 39.9342 & 51 \\
\hline 116.4773 & 39.9731 & 51 \\
\hline 116.3647 & 39.9022 & 51 \\
\hline 116.5386 & 40.0303 & 51 \\
\hline 116.5614 & 40.0355 & 51 \\
\hline 116.5671 & 40.0345 & 51 \\
\hline 130.2822 & 47.3069 & 51 \\
\hline 116.5448 & 40.0331 & 51 \\
\hline 116.5018 & 39.9195 & 51 \\
\hline 116.4712 & 39.8774 & 50 \\
\hline 116.4164 & 39.9588 & 50 \\
\hline 116.4898 & 39.9182 & 50 \\
\hline 116.3666 & 39.9542 & 50 \\
\hline 116.3273 & 39.974 & 50 \\
\hline 116.3231 & 39.9335 & 49 \\
\hline 116.3733 & 39.9941 & 49 \\
\hline 116.4384 & 39.9713 & 49 \\
\hline 116.3763 & 40.0101 & 48 \\
\hline 116.4468 & 39.8666 & 48 \\
\hline 116.4968 & 39.9147 & 47 \\
\hline 116.4406 & 39.997 & 47 \\
\hline 116.46 & 39.9428 & 47 \\
\hline 130.2748 & 47.2994 & 47 \\
\hline 116.3613 & 39.9447 & 47 \\
\hline 116.591 & 40.092 & 47 \\
\hline 116.356 & 40.034 & 47 \\
\hline 116.3384 & 40.0535 & 47 \\
\hline 115.686 & 37.7388 & 47 \\
\hline 116.3122 & 39.9699 & 46 \\
\hline 116.459 & 39.9831 & 46 \\
\hline 116.4604 & 39.8735 & 46 \\
\hline 116.418 & 39.9739 & 46 \\
\hline 116.322 & 39.8976 & 46 \\
\hline 116.4037 & 39.9771 & 46 \\
\hline 116.3602 & 40.0176 & 46 \\
\hline 116.4085 & 39.9515 & 46 \\
\hline 116.3972 & 39.9535 & 46 \\
\hline 116.3151 & 39.9487 & 46 \\
\hline 116.3441 & 39.9058 & 45 \\
\hline 116.3663 & 40.0268 & 45 \\
\hline 130.272 & 47.2883 & 45 \\
\hline
\end{tabular}




\begin{tabular}{|c|c|c|}
\hline 120.3032 & 30.4392 & 45 \\
\hline 116.5904 & 40.079 & 45 \\
\hline 116.4724 & 39.9333 & 45 \\
\hline 129.6056 & 44.5991 & 45 \\
\hline 116.4423 & 39.9592 & 44 \\
\hline 116.3284 & 40.0702 & 44 \\
\hline 116.3623 & 39.9349 & 44 \\
\hline 116.4393 & 39.9335 & 44 \\
\hline 116.3448 & 39.9661 & 44 \\
\hline 116.33 & 40.0689 & 44 \\
\hline 116.4199 & 39.9949 & 44 \\
\hline 116.5145 & 39.9115 & 44 \\
\hline 116.3569 & 39.9064 & 43 \\
\hline 116.446 & 39.8975 & 43 \\
\hline 116.3918 & 39.9795 & 43 \\
\hline 116.3372 & 39.994 & 43 \\
\hline 116.5927 & 40.0608 & 43 \\
\hline 116.2686 & 39.9312 & 43 \\
\hline 116.3559 & 39.9296 & 43 \\
\hline 116.6251 & 40.0529 & 42 \\
\hline 116.2815 & 39.9222 & 42 \\
\hline 116.3447 & 39.9877 & 42 \\
\hline 116.5972 & 40.0565 & 42 \\
\hline 116.443 & 39.942 & 42 \\
\hline 116.2858 & 39.9173 & 42 \\
\hline 116.3655 & 39.9238 & 42 \\
\hline 116.3013 & 39.9109 & 42 \\
\hline 116.355 & 39.8583 & 42 \\
\hline 116.4936 & 39.9213 & 42 \\
\hline 116.469 & 39.8738 & 42 \\
\hline 116.3556 & 40.0373 & 42 \\
\hline 116.3147 & 39.9905 & 42 \\
\hline 116.6282 & 40.0506 & 42 \\
\hline
\end{tabular}

\section{Copyrights}

Copyright for this article is retained by the author(s), with first publication rights granted to the journal.

This is an open-access article distributed under the terms and conditions of the Creative Commons Attribution license (http://creativecommons.org/licenses/by/4.0/). 